\title{
Percepción de estudiantes de pedagogía en relación a las oportunidades para el desarrollo de prácticas generativas en su formación
}

\author{
Teacher candidates' perceptions of opportunities to develop core practices \\ in a teacher education program
}

\author{
Magdalena Muller ${ }^{a}$, Pilar Álamos $^{b}$, Lorena Meckes $^{c}$, Anita Sanyal $^{d}$, Pilar Cox ${ }^{e}$ \\ a Pontificia Universidad Católica de Chile. Correo electrónico: mbmuller@uc.cl

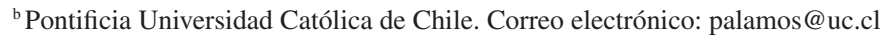 \\ cCentro de Estudios de Políticas y Prácticas en Educación. Correo electrónico: 1meckes@uc.cl \\ ${ }^{d}$ Pontificia Universidad Católica de Chile. Correo electrónico: asanyal@ uc.cl

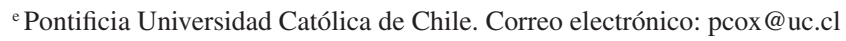

\begin{abstract}
RESUMEN
El desafío de integrar teoría y práctica en el curriculum de formación inicial de profesores es un tema relevante en la agenda de investigación en este campo. Se presentan los resultados de un cuestionario sobre la percepción de estudiantes en relación a las oportunidades para el desarrollo de prácticas generativas en su trayectoria formativa, que fue diseñado con el propósito de monitorear un proceso de rediseño curricular que apunta a poner la práctica como eje de la formación y a recoger evidencia para su ajuste. El instrumento pregunta a 1.294 estudiantes de cursos disciplinares, didácticas y prácticas acerca de su percepción sobre la coherencia y las distintas oportunidades de aproximación a la práctica que han tenido. Se hace una comparación entre cursos. Menos de la mitad de los estudiantes (porcentaje $\leq 50 \%$ ) señaló contar con oportunidades para ensayar prácticas generativas en los cursos de didáctica y práctica en la universidad.
\end{abstract}

Palabras clave: prácticas generativas, formación inicial de profesores, percepción de estudiantes, coherencia, aproximaciones a la práctica

\section{ABSTRACT}

The challenge of integrating theory and practice in the curriculum of initial teacher training is an important issue in the research agenda in this field. The results of a questionnaire on perceptions of students in relation to opportunities for the development of high leverage practices in their education programs are discussed. The instrument was designed with the purpose of monitoring a process of curriculum redesign aimed at putting practice as the core of the training process and to look for evidence for adjustment of the change process. The instrument was applied to 1,294 students of disciplinary, methods and field experience courses and asked their perception about coherence and opportunities to different approximations to practice in the courses. The results between courses are compared. Less than half of students (percentage $\leq 50 \%$ ) indicated having opportunities to rehearse high leverage practices in methods and field experience courses at the university.

Keywords: high leverage practices, pre-service teacher education, students' perceptions, coherence, approximations to practice 
Estudios Pedagógicos XLII, Nº 4: 145-163, 2016

PERCEPCIÓN DE ESTUDIANTES DE PEDAGOGÍA EN RELACIÓN A LAS OPORTUNIDADES PARA EL DESARROLLO DE PRÁCTICAS GENERATIVAS EN SU FORMACIÓN

\section{INTRODUCCIÓN}

Las crecientes demandas de una educación de calidad han volcado la atención hacia el rol protagónico de los profesores en los aprendizajes de sus estudiantes (River \& Sanders, 1996; Hanushek, 1992) y hacia un fortalecimiento de su formación inicial (OCED, 2005; Darling-Hammond, 2000). Tanto desde las políticas públicas (OREALC/UNESCO, 2013; Musset, 2010) como desde intervenciones específicas (Davis \& Boerst, 2014; Hammerness, 2006), se observan esfuerzos por mejorar la formación inicial que reciben los profesores, como un medio estratégico para mejorar la calidad de la educación.

La mayoría de los programas de formación inicial docente se caracterizan por ser muy teóricos; entendiendo por ello abstractos, generales y con un foco en las creencias y el conocimiento. La evidencia da cuenta de que los futuros profesores tienden a aprender conocimientos y desarrollar habilidades analíticas en torno a la enseñanza en desmedro de desempeños específicos para la enseñanza en la sala de clases (Ball \& Forzani, 2009; Grossman, Compton, Igra, Ronfeldt, Shahan \& Williamson, 2009; Darling-Hammond, Hammerness, Grossman, Rust \& Shulman, 2005).

¿Por qué ha sido tan teórica la formación inicial de los profesores? Entre otras causas, la falta de un consenso o de un cuerpo de conocimientos respecto a qué es una buena enseñanza ha contribuido a la dificultad para identificar qué es lo que un profesor debe aprender durante su formación inicial y, por ende, ser capaz de realizar profesionalmente tras su egreso (Mc Donald, Kazemi \& Schneider, 2013). En relación con ello, un estudio de Grossman, Compton, Igra, Ronfeldt, Shahan \& Williamson (2009), comparó la formación de profesores con la de otras profesiones que también trabajan directamente con personas psicólogos y sacerdotes- y halló que, entre las tres, los profesores son quienes tienen menos oportunidades para aproximarse en forma específica a la práctica propia de su ejercicio profesional. De ello se desprende la atención que debe ponerse tanto en identificar aquello que es propio de la práctica pedagógica como en el desarrollo de un currículum anclado en dichas prácticas en la formación inicial docente.

El objetivo de este estudio es reportar la percepción de coherencia y de oportunidades de aprendizaje para el desarrollo de prácticas generativas de estudiantes de un Programa de Educación General Básica, que está en un proceso de innovación, que implica el rediseño de los cursos: 1) disciplinares (Lenguaje, Matemática, Historia y Ciencias), 2) didácticas y 3) prácticas. El foco del rediseño es avanzar hacia un curriculum basado en la práctica. Con el fin de monitorear aspectos claves del proceso de cambio, se elaboró un instrumento que permite levantar la percepción de los estudiantes en relación a elementos específicos de su formación. Se presentan los indicadores que se relacionan con programas de formación docente efectivo, tales como, la coherencia de éste tanto a nivel estructural como conceptual, y se especifica qué se espera que aprendan los estudiantes durante su formación en el programa, específicamente, el concepto de prácticas generativas (High Leverage Practices). 


\section{ANTECEDENTES TEÓRICOS Y EMPÍRICOS}

\subsection{LA COHERENCIA COMO INDICADOR DE PROGRAMAS EFECTIVOS DE FORMACIÓN INICIAL DOCENTE}

Al diseñar un programa de formación inicial docente, la primera pregunta que emerge es ¿qué se considera una buena enseñanza? En otras palabras, ¿qué se espera de dichos profesores una vez que terminen su proceso formativo? Esta interrogante alude a la visión del programa, la cual incorpora dimensiones como el rol del profesor, la naturaleza de la enseñanza-aprendizaje y la misión de la escuela en democracia (Darling-Hammond et al., 2005).

En relación a lo anterior, la revisión de investigaciones aporta evidencia en favor de que los programas de formación inicial docente son más efectivos cuando tienen un alto grado de coherencia en su visión (Darling-Hammond et al., 2005). Esta coherencia alude tanto a aspectos estructurales como conceptuales. Desde un punto de vista estructural, se refiere a la organización del curriculum, considerando tanto el alineamiento entre los cursos como dentro de los mismos. Estos significados se plasman, por ejemplo, en la estructura y alineación de los cursos, en su secuencia a lo largo del programa de formación, en su relación con los perfiles de egreso, entre otros. A nivel conceptual, alude a una visión compartida acerca de la enseñanza y al vínculo intencionado entre teoría y práctica en el curriculum (Hamerness, 2006). Esta coherencia se manifiesta en el desarrollo de significados compartidos entre los académicos del programa, que expliquen, justifiquen y creen consenso en torno a qué implica una buena enseñanza. Ahora bien, el aprender acerca de qué es una buena enseñanza no resulta suficiente. También se requiere que los profesores en formación tengan oportunidades para practicar y actuar dicha visión (Hammerness \& Klette, 2015).

En síntesis, el desarrollo de la experticia en el campo pedagógico requiere de los programas de formación una visión coherente respecto de qué se entiende por una enseñanza de calidad, y que dicha visión informe la organización de las oportunidades de aprendizaje a lo largo de la trayectoria formativa. Por ello, tanto la coherencia como la aproximación a la práctica son relevadas como indicadores de los programas que permiten formar profesores con los conocimientos, habilidades y disposiciones necesarias para enseñar más eficazmente a sus estudiantes.

\subsection{APRENDIZAJE DE PRÁCTICAS GENERATIVAS EN LA FORMACIÓN INICIAL DOCENTE}

Para tener una visión coherente dentro de un programa de formación se hace imprescindible definir lo que los estudiantes de pedagogía deben aprender, para poder enseñar a una diversidad de estudiantes en contextos variados. Estos desempeños han sido objeto de estudio en la última década y se conocen como prácticas nucleares, esenciales o generativas (core practices/ high leverage practices). En este estudio se hace referencia a estas prácticas como prácticas generativas, ya que este concepto alude a que son prácticas que debiese dominar un novato, que tienen el potencial de generar desempeños más complejos a medida que se integran entre sí y que se va teniendo más experiencia en la sala de clases. Estas prácticas generativas buscan descomponer (Grossman et al., 2009) o desglosar la práctica pedagógica, con el fin de que puedan ser aprendidas y evaluadas en 
Estudios Pedagógicos XLII, N 4: 145-163, 2016

PERCEPCIÓN DE ESTUDIANTES DE PEDAGOGÍA EN RELACIÓN A LAS OPORTUNIDADES PARA EL DESARROLLO DE PRÁCTICAS GENERATIVAS EN SU FORMACIÓN

la trayectoria formativa de los profesores en formación. Si bien, actualmente en Chile, se cuenta con estándares orientadores para la formación inicial, estos son más bien genéricos. Por ejemplo, un estándar pedagógico es: “conoce a los estudiantes de Educación Básica y sabe cómo aprenden". Uno de los indicadores para este estándar es: "es responsable del aprendizaje de todos los estudiantes, para ello conoce y diseña estrategias que promuevan el desarrollo de cada uno de ellos". Aquí surgen preguntas para los formadores de profesores como: ¿cuáles son las partes constitutivas de este estándar?, ¿qué tendría que practicar un novato para lograrlo? En este contexto las prácticas generativas, al ser desempeños propios de la enseñanza-aprendizaje susceptibles de ser identificados, nombrados, enseñados y evaluados (Ball \& Forzani, 2009), se transforman en un recurso clave para el logro de estos estándares.

Un modelo de definición de prácticas generativas es el elaborado por la Universidad de Michigan para su programa de formación inicial de profesores de educación general básica. Este modelo aborda aspectos relacionados con: 1) la planificación, como por ejemplo, "poner metas de aprendizaje de corto y mediano plazo para los alumnos alineadas con referentes externos"; 2) la interacción en la sala de clases, como por ejemplo, "elicitar e interpretar el pensamiento de cada alumno"; 3) la organización de la sala de clases, como por ejemplo, "facilitar y conducir el trabajo en grupo pequeño"; 4) la evaluación, como por ejemplo, "dar retroalimentación oral y escrita a los estudiantes sobre su trabajo"; 5) la comunicación con distintitos actores, como por ejemplo, "comunicarse con padres y apoderados sobre sus alumnos"; y 6) la reflexión, como por ejemplo, “analizar la propia práctica con el propósito de mejorarla" (Davis \& Boerst, 2014).

Tradicionalmente, los profesores en formación tienen que desplegar sus recursos pedagógicos en los contextos de máxima complejidad, es decir, en salas de clases reales con el grupo completo de estudiantes. La idea de tener aproximaciones sucesivas a la práctica planteada por Grossman et al. (2009), es preparar a los futuros profesores en contextos más controlados con tareas menos auténticas, lo que denominan como una dimensión representacional, en una primera instancia, para que progresivamente se expongan a experiencias de mayor autenticidad y complejidad. De acuerdo con esto, el brindar a los profesores en formación oportunidades para ensayar y simular estos desempeños, y aproximarse gradualmente a ellos hasta llegar a ponerlos en acto (enactment) en contextos reales para luego analizar cómo mejorar, ha sido, precisamente, el objetivo último de identificar y conceptualizar las prácticas generativas (Mc Donald, Kazemi \& Schneider, 2013; Center to Support Excellence on Teaching - CSET, s/f).

\subsection{REDISEÑO DE PROGRAMAS CON FOCO EN LA INTEGARCIÓN DE TEORÍA Y PRÁCTICA}

Si bien la evidencia teórica y empírica plantea la necesidad de que los programas y cursos de la formación docente avancen hacia un currículum basado en la práctica, se sabe menos acerca de cómo diseñar un currículum de estas características (Davis \& Boerst, 2014; Hammerness, 2006). En este sentido, existe escasa evidencia local que reporte cómo programas de carácter más bien teórico pueden transitar hacia un curriculum basado en la práctica pedagógica. Este estudio se sitúa en un proceso de rediseño de cursos que se concentró, en su primera fase, en los tres tipos de cursos que conforman el núcleo formativo del programa: los cursos de (1) disciplina, (2) didáctica y (3) práctica. Los cursos de disciplina se refieren a aquellos en que los futuros profesores aprenden contenidos 
específicos de la asignatura, y que son dictados por académicos expertos en la materia en las respectivas facultades disciplinares. Por su parte, los cursos de didácticas son dictados por académicos que forman parte de la Unidad Académica de Educación y aluden a cursos pedagógicos-disciplinares, cuyo foco es la enseñanza de la asignatura en el contexto escolar. Por último, los cursos de práctica son aquellos de experiencia clínica en colegios, a través de los cuales los profesores en formación se aproximan de manera más directa a la práctica, con alumnos en la sala de clases acompañados tanto por un profesor de la Universidad como por un profesor del contexto escolar. Si bien el rediseño abordó tanto el Programa de Educación de Párvulos, como los de Educación Media y Educación General Básica, este estudio se focaliza en la intervención realizada en este último.

El modelo de cambio que sustenta este rediseño curricular considera, entre otros, los tres conceptos claves para la comprensión de las pedagogías de la práctica en el aprendizaje profesional descritos más arriba: representación, descomposición y aproximación a la práctica (Grossman et al., 2009). Es así como, en los cursos disciplinares, el foco de intervención en relación con las prácticas generativas se centra en la representación de la práctica, a nivel de observación de modelos, lo que implica que los estudiantes tengan oportunidades de ver a sus profesores utilizando determinadas prácticas generativas en su docencia universitaria. En la didáctica se incluye tanto la dimensión representativa como la de tener oportunidades de ponerlas en acto a nivel de ensayo o simulación. Y en los cursos de práctica, el énfasis está en incluir, además de oportunidades a nivel representacional y de ensayo, oportunidades de experiencia directa en contextos reales con distinto nivel de complejidad, dado por la cantidad de alumnos con los que se trabaje (un estudiante, grupo pequeño, grupo completo).

En este contexto de cambio surge la necesidad de monitorear la implementación de los cursos rediseñados. Hasta ahora la única fuente de información respecto a los cursos es una encuesta en la que los estudiantes evalúan principalmente la docencia universitaria en forma genérica. Es por esto que se considera necesario diseñar un instrumento que permita monitorear los cambios diseñados en forma más específica. Uno de los instrumentos diseñados con este fin es un cuestionario que recoge las percepciones de los estudiantes en relación a las oportunidades de aprendizaje percibidas en distintas instancias de su trayectoria formativa (Müller, en prensa).

\section{OBJETIVOS Y PREGUNTAS DE INVESTIGACIÓN}

El objetivo de este estudio es analizar la percepción de coherencia en la formación y de oportunidades de aprendizaje para el desarrollo de prácticas generativas de estudiantes de un Programa de Educación General Básica en cursos: 1) disciplinares (Lenguaje, Matemática, Historia y Ciencias), 2) didáctica y 3) prácticas y comparar esta percepción entre cursos.

Así, las preguntas de investigación que guían el estudio son las siguientes:

1. ¿Cuáles son las diferencias entre los cursos disciplinares, didácticos y prácticos en la coherencia percibida por los estudiantes?

2. ¿Cuáles son las diferencias entre cursos disciplinares, didácticos y prácticos en la percepción de oportunidades para estar expuesto a modelos de prácticas generativas? 
3. ¿Cuáles son las diferencias entre cursos didácticos y prácticos en relación a oportunidades para ver representaciones de práctica (análisis de casos y modelamiento/demostraciones)?

4. ¿Cuál es la diferencia entre cursos didácticos y prácticos en las oportunidades de aprendizaje percibidas por los estudiantes para ensayar prácticas generativas?

5. ¿Cuáles son las diferencias en las experiencias directas de los estudiantes según el tipo de práctica realizada?

\section{MÉTODOS}

\subsection{PARTICIPANTES}

Los participantes del estudio fueron estudiantes de un programa de formación en Pedagogía General Básica de una institución universitaria tradicional, en el año 2014. Dicho programa tiene una duración de 10 semestres y conduce al título profesional de Profesor en Educación General Básica. Si bien la Universidad ofrece otros programas de formación pedagógica, anteriormente mencionados, para este estudio se escogió el de Educación General Básica por concentrar un mayor número de estudiantes y cursos, permitiendo acceder a un mayor tamaño muestral.

Tal como se aprecia en la tabla 1, participaron 1.294 estudiantes, 864 en el primer semestre y 430 en el segundo. Todos los estudiantes, en su mayoría mujeres de más de 20 y menos de 25 años, dieron su consentimiento para participar en el estudio.

Tabla 1. Distribución de la muestra según género, edad y tipo de curso

\begin{tabular}{llccc}
\hline & & Primer semestre & Segundo semestre & Total \\
\hline \multirow{3}{*}{ Género } & Femenino & 801 & 398 & 1199 \\
& Masculino & 63 & 32 & 95 \\
& Datos perdidos & 0 & 0 & 0 \\
\hline \multirow{2}{*}{ Total } & & 864 & 430 & 1294 \\
\hline \multirow{5}{*}{ Edad } & 20 o menos años & 397 & 137 & 534 \\
& Más de 20 y menos de 25 años & 404 & 253 & 657 \\
& 25 años o más & 58 & 38 & 96 \\
Total & Datos perdidos & 5 & 2 & 7 \\
\hline \multirow{4}{*}{ Tipo de curso } & Didáctico & 864 & 430 & 1294 \\
& Práctico & 442 & 0 & 442 \\
& & 341 & 192 & 533 \\
Total & & 81 & 238 & 319 \\
\hline
\end{tabular}




\subsection{INSTRUMENTO}

El instrumento utilizado en este estudio corresponde a un cuestionario de percepción de los estudiantes respecto de sus oportunidades de aprendizaje. Dicho cuestionario indaga la percepción de una visión coherente del proceso de enseñanza-aprendizaje y de las oportunidades para aprender un conjunto de prácticas generativas en los distintos cursos. Ambos tipos de oportunidades de aprendizaje, coherencia y prácticas generativas constituyen dos de los focos abordados en el mencionado rediseño.

Para elaborar el cuestionario se tomó como punto de partida el instrumento diseñado en el marco del estudio Coherence and Assignments in Teacher Education [CATE] (Hammerness \& Klette, 2015). Este cuestionario, diseñado para cursos didácticos, se compone de 36 ítems que indagan en las percepciones de los estudiantes respecto de sus oportunidades de aprendizaje en la formación inicial. Por ejemplo, “ ¿En qué medida tuviste oportunidades para examinar muestras de trabajos auténticos de alumnos en tus cursos didácticos?”. Los 36 ítems que conforman este cuestionario se agrupan en tres indicadores de calidad de la formación inicial docente: 1) la existencia de una visión compartida de la enseñanza (5 ítems); 2) la coherencia de dicha visión en el programa (14 ítems); y 3) la aproximación a la práctica pedagógica (17 ítems). Para cada ítem los estudiantes deben marcar una de cuatro opciones en una escala de Likert. Si bien las dimensiones de coherencia y aproximación eran una buena base para la elaboración del instrumento, el cuestionario CATE presentaba dos limitaciones para cumplir los objetivos del presente estudio. Primero, no tenía un foco explícito en prácticas generativas específicas y las aproximaciones para aprenderlas, que son un aspecto a indagar para la transición hacia un currículum anclado en la práctica. Segundo, el cuestionario CATE fue diseñado para los cursos didácticos y no todos sus ítems eran pertinentes o suficientes para los cursos disciplinares y de práctica.

Del cuestionario CATE se mantienen 5 ítems relacionados con coherencia, que aluden a la visión sobre la enseñanza y el aprendizaje entre cursos, a la lógica de progresión entre éstos y al conocimiento del profesor con respecto a la relación entre cursos tanto a nivel de actividades como de ideas. Por otra parte, el cuestionario ajustado permite distinguir dos aproximaciones con distinto nivel de autenticidad en relación al aprendizaje de las prácticas generativas: (i) una representacional, que incluye tanto estar expuesto a modelos como analizar casos y ver demostraciones, y (ii) otra de poner en acto (enacting), que comprende tanto ensayos y simulaciones, como la exposición directa a la práctica. Cabe destacar que ensayo, en este estudio, no refiere exclusivamente a ensayar las interacciones con alumnos, sino también a ensayar la planificación y la evaluación.

El cuestionario se diseñó en base al modelo de cambio antes descrito por lo que consta de 3 versiones, una para cada tipo de curso: disciplinares, didácticos y prácticos. Como muestra la tabla 2 las oportunidades de percibir coherencia y estar expuesto a prácticas generativas en los cursos, son consultadas en los tres tipos de intervenciones: disciplinar, didáctica y práctica. Las experiencias para representarse la práctica a través de demostraciones o modelamiento y análisis de casos, y para ensayar, sólo se preguntan en los cursos de didáctica y práctica. Por último, las oportunidades para tener experiencias directas en aula sólo fue incluida en los cuestionarios de los cursos de práctica. 
Tabla 2. Lógica del diseño del cuestionario

\begin{tabular}{|c|c|c|c|c|c|}
\hline \multirow{2}{*}{$\begin{array}{l}\text { Oportunidades } \\
\text { de aprendizaje }\end{array}$} & & & \multicolumn{3}{|c|}{ Tipo de curso } \\
\hline & & & Disciplinares & Didácticos & Prácticos \\
\hline Coherencia & $\begin{array}{l}\text { 1. Experimentar } \\
\text { coherencia }\end{array}$ & & $\mathrm{x}$ & $\mathrm{x}$ & $\mathrm{x}$ \\
\hline \multirow{4}{*}{$\begin{array}{l}\text { Aprender } \\
\text { prácticas } \\
\text { generativas }\end{array}$} & \multirow{2}{*}{$\begin{array}{l}\text { 1. Nivel } \\
\text { Representacional }\end{array}$} & $\begin{array}{l}\text { 1.1. Estar expuesto a } \\
\text { practicas generativas }\end{array}$ & $\mathrm{x}$ & $\mathrm{x}$ & $\mathrm{x}$ \\
\hline & & $\begin{array}{l}\text { 1.2. Análisis de casos } \\
\text { y modelamiento/ } \\
\text { demostraciones }\end{array}$ & & $\mathrm{x}$ & $\mathrm{x}$ \\
\hline & \multirow{2}{*}{$\begin{array}{l}\text { 2. Nivel de } \\
\text { ensayo }\end{array}$} & 2.1. Simulación & & $\mathrm{x}$ & $\mathrm{x}$ \\
\hline & & $\begin{array}{l}\text { 2.2. Experiencia } \\
\text { directa }\end{array}$ & & & $\mathrm{x}$ \\
\hline
\end{tabular}

La mayor parte de las preguntas, que conforman la primera parte del cuestionario, son ítems tipo escala Likert. Los estudiantes marcan una de entre 4 opciones que van desde "no tuve oportunidades" hasta "tuve muchas oportunidades". Cabe destacar que también existe una opción llamada "No aplica al curso". En la segunda parte del cuestionario se incluyen algunas preguntas acerca de la preparación de la enseñanza e ítems en relación a las oportunidades de tener experiencia directa. En este caso, se pide a los estudiantes que respondan en una primera instancia si han tenido o no la experiencia, para luego en una segunda instancia, en caso de dar una respuesta afirmativa, pedirles que marquen el nivel de aproximación que han tenido con dicha práctica: "ensayando en clases en la Universidad", "practicando con un niño", "practicando con un grupo pequeño" y/o "practicando con el curso completo".

Respecto a las propiedades métricas de la primera parte del instrumento, en su primera aplicación (primer semestre del 2014), se evaluó su consistencia interna mediante el estadístico alfa de Cronbach. Previo a ello, mediante la prueba MCAR de Little, se comprobó el supuesto de que los datos están perdidos completamente al azar, y se trabajaron los datos perdidos mediante el algoritmo de maximización esperada. Como se observa en la tabla 3, las diferentes versiones muestran niveles aceptables de consistencia, lo que aporta evidencia a favor de su uso.

Tabla 3. Consistencia Interna de las diferentes versiones del cuestionario

\begin{tabular}{lc}
\hline \multicolumn{1}{c}{ Tipo de curso } & Alfa de Cronbach \\
\hline Disciplinares & 0,92 \\
Didácticos & 0,95 \\
Prácticos & 0,92 \\
\hline
\end{tabular}




\subsection{PROCEDIMIENTO}

Las tres versiones preliminares del instrumento se sometieron a una evaluación piloto el segundo semestre del año 2013. El pilotaje consistió en la aplicación del cuestionario a una muestra de 426 estudiantes del programa de Pedagogía General Básica.

Del pilotaje se recogieron las reacciones de los encuestados -como facilidad, aburrimiento y fatiga-, se ajustaron las instrucciones para presentar los ítems y se suprimieron algunos cuya tasa de respuesta "no aplica" fue muy alta. Como parte de este proceso, y dada la extensión del cuestionario, se optó por dividirlo en dos partes.

Las versiones finales de la primera y segunda parte de los cuestionarios fueron aplicadas en forma presencial a la muestra de participantes al finalizar el primer y segundo semestre del año 2014, respectivamente. Las instrucciones para responder el instrumento se dieron por escrito, de manera que el instrumento fuera auto-aplicado. Con esto se buscó garantizar la confiabilidad de los resultados y disminuir en lo posible cualquier variable interviniente que pudiera afectar las respuestas de los estudiantes. La aplicación del instrumento se realizó durante los mismos cursos y tuvo una duración aproximada de 25 a 30 minutos.

\subsection{ANÁLISIS DE DATOS}

Para tener una visión panorámica de las respuestas, se calculó la media y la desviación estándar para cada ítem, y se contrastó las respuestas mínima y máxima con el mínimo y máximo teóricos. Para ver si existían diferencias significativas entre los tipos de cursos, se realizaron pruebas t o análisis de la varianza (ANOVA) de un factor y contrastes post hoc. Se utilizó la prueba HSD de Tukey, pues esta tenía la ventaja de permitir hacer todas las posibles comparaciones entre tipos de cursos y, así, conocer dónde se encontraban las diferencias. Para responder a las preguntas de ensayo en los cursos didácticos y prácticos, se analizaron las frecuencias de respuesta para cada ítem.

\section{RESULTADOS}

\subsection{OPORTUNIDADES DE APRENDIZAJE PERCIBIDAS POR LOS ESTUDIANTES: COHERENCIA}

¿Cuáles son las diferencias entre los cursos disciplinares, didácticos y prácticos en la coherencia percibida por los estudiantes?

Los resultados descriptivos muestran que todos los ítems de coherencia alcanzan el mínimo y máximo teórico, 1 y 4 respectivamente, y que las medias se concentran en un rango entre 2,41 y 2,95. Solo uno de los ítems, "conexión de conceptos e ideas dentro de un mismo curso", alcanza una media superior de 3,31 (DE =0,80).

Al comparar las medias en la percepción de coherencia entre los distintos tipos de cursos, los análisis (ANOVA de un solo factor o Welch, dependiendo si se mantiene el supuesto de homogeneidad de las varianzas o no) confirmaron diferencias significativas $(\mathrm{p}<0,05)$ en todos los ítems.

Las comparaciones post hoc, usando la prueba de Tukey HSD, permiten observar que la percepción de coherencia para los cursos disciplinares es significativamente menor que 
para los cursos didácticos y prácticos $(\mathrm{p}<0,05)$. Solo para uno de los ítems, acerca de la conexión de ideas y conceptos entre clases del mismo curso, no se confirmaron diferencias significativas entre los cursos disciplinares $(M=3,20 ; D E=0,82)$ y prácticos $(M=3,34$; $\mathrm{DE}=0,72)$. Sin embargo, para este ítem sí se observó una diferencia significativa al contrastarlo con los cursos de didáctica $(\mathrm{M}=3,44 ; \mathrm{DE}=0,79)$. Para ninguno de los ítems se observan diferencias significativas en la percepción de coherencia entre los cursos de didáctica y de práctica.

Tabla 4. Prueba de Tukey HSD para coherencia entre los diferentes tipos de curso

\begin{tabular}{|c|c|c|c|c|}
\hline Pregunta & Tipo de curso & $\begin{array}{l}\text { Diferencia } \\
\text { promedio }\end{array}$ & $\begin{array}{l}\text { Error } \\
\text { estándar }\end{array}$ & $\mathrm{P}$ \\
\hline \multirow{3}{*}{$\begin{array}{l}\text { Apreciar en el curso una visión } \\
\text { sobre la enseñanza y aprendizaje } \\
\text { que es coherente con la que he } \\
\text { experimentado en otros cursos de } \\
\text { esta área disciplinaria }\end{array}$} & Disciplinar-Didáctica & $-0,82 * *$ & 0,06 & 0,00 \\
\hline & Disciplinar - Práctica & $-0,66 * *$ & 0,11 & 0,00 \\
\hline & Didáctica - Práctica & 0,16 & 0,11 & 0,29 \\
\hline \multirow{3}{*}{$\begin{array}{l}\text { Conectar ideas y conceptos entre } \\
\text { clases del mismo curso, y apreciar } \\
\text { una lógica de progresión en su } \\
\text { organización }\end{array}$} & Disciplinar-Didáctica & $-0,25 * *$ & 0,06 & 0,00 \\
\hline & Disciplinar - Práctica & $-0,14$ & 0,10 & 0,29 \\
\hline & Didáctica - Práctica & 0,10 & 0,10 & 0,56 \\
\hline \multirow{3}{*}{$\begin{array}{l}\text { Profundizar el estudio y } \\
\text { comprensión de conceptos o } \\
\text { lecturas ya vistas en otros cursos } \\
\text { de la misma disciplina o área }\end{array}$} & Disciplinar-Didáctica & $-0,97 * *$ & 0,07 & 0,00 \\
\hline & Disciplinar - Práctica & $-1,10^{* *}$ & 0,11 & 0,00 \\
\hline & Didáctica - Práctica & $-0,13$ & 0,11 & 0,49 \\
\hline \multirow{3}{*}{$\begin{array}{l}\text { Ver que el profesor(a) sabía lo } \\
\text { que estábamos haciendo en otros } \\
\text { cursos (ej. Tareas, lecturas, ideas } \\
\text { claves y evaluaciones) }\end{array}$} & Disciplinar-Didáctica & $-0,66 * *$ & 0,08 & 0,00 \\
\hline & Disciplinar - Práctica & $-0,39 *$ & 0,13 & 0,01 \\
\hline & Didáctica - Práctica & 0,27 & 0,13 & 0,10 \\
\hline \multirow{3}{*}{$\begin{array}{l}\text { Ver que el profesor de este curso } \\
\text { conectaba explícitamente ideas } \\
\text { del curso con las ideas vistas o } \\
\text { discutidas en otro(s) curso(s) }\end{array}$} & Disciplinar-Didáctica & $-0,94 * *$ & 0,07 & 0,00 \\
\hline & Disciplinar - Práctica & $-0,78 * *$ & 0,12 & 0,00 \\
\hline & Didáctica - Práctica & 0,15 & 0,12 & 0,39 \\
\hline
\end{tabular}



GENERATIVAS

\subsubsection{Nivel representacional}

\subsubsection{Exposición a prácticas generativas en la docencia universitaria}

¿Cuáles son las diferencias entre cursos disciplinares, didácticos y prácticos en la percepción de oportunidades de aprendizaje para estar expuesto a practicas generativas en docencia universitaria?

Todos los ítems de exposición a prácticas generativas alcanzan el mínimo y máximo teóricos y sus medias superan los 3 puntos, concentrándose en un rango entre 3,15 y 3,27. Una síntesis de diferencias observadas por tipo de curso se presenta en la tabla 5:

Tabla 5. Prueba de Tukey HSD para modelamiento entre los diferentes tipos de curso

\begin{tabular}{|c|c|c|c|c|}
\hline Pregunta & Tipo de curso & $\begin{array}{l}\text { Diferencia } \\
\text { promedio }\end{array}$ & $\begin{array}{l}\text { Error } \\
\text { estándar }\end{array}$ & $\mathrm{P}$ \\
\hline \multirow{3}{*}{$\begin{array}{l}\text { Ver que el profesor usaba } \\
\text { estratégicamente representaciones, } \\
\text { modelos o ejemplos que facilitaron } \\
\text { mi comprensión de los conceptos } \\
\text { trabajados en el curso }\end{array}$} & Disciplinar-Didáctica & 0,00 & 0,06 & 0,99 \\
\hline & Disciplinar - Práctica & $0,30^{*}$ & 0,10 & 0,01 \\
\hline & Didáctica - Práctica & $0,29 *$ & 0,10 & 0,01 \\
\hline \multirow{3}{*}{$\begin{array}{l}\text { Ver que el profesor del curso } \\
\text { explicitaba sus propios procesos } \\
\text { de pensamiento al resolver } \\
\text { un problema o hacer una } \\
\text { demostración en clases }\end{array}$} & Disciplinar-Didáctica & 0,08 & 0,06 & 0,41 \\
\hline & Disciplinar - Práctica & $0,43 * *$ & 0,11 & 0,00 \\
\hline & Didáctica - Práctica & $0,35^{* *}$ & 0,11 & 0,00 \\
\hline \multirow{3}{*}{$\begin{array}{l}\text { Ver que el profesor facilitaba } \\
\text { discusiones en clases que me } \\
\text { permitían aprender de los } \\
\text { argumentos de mis compañeros y } \\
\text { expresar mis argumentos frente a } \\
\text { un problema o tema }\end{array}$} & Disciplinar-Didáctica & $-0,48^{* *}$ & 0,07 & 0,00 \\
\hline & Disciplinar - Práctica & $-0,64 * *$ & 0,11 & 0,00 \\
\hline & Didáctica - Práctica & $-0,16$ & 0,11 & 0,34 \\
\hline \multirow{3}{*}{$\begin{array}{l}\text { Realizar tareas y actividades } \\
\text { propuestas en clases que eran } \\
\text { desafiantes y apuntaban a metas } \\
\text { específicas del curso }\end{array}$} & Disciplinar-Didáctica & $-0,38 * *$ & 0,06 & 0,00 \\
\hline & Disciplinar - Práctica & $-0,22$ & 0,10 & 0,06 \\
\hline & Didáctica - Práctica & 0,16 & 0,10 & 0,26 \\
\hline
\end{tabular}


Estudios Pedagógicos XLII, $\mathrm{N}^{\circ}$ 4: 145-163, 2016

PERCEPCIÓN DE ESTUDIANTES DE PEDAGOGÍA EN RELACIÓN A LAS OPORTUNIDADES PARA EL DESARROLLO DE PRÁCTICAS GENERATIVAS EN SU FORMACIÓN

En los cursos disciplinares y didácticos, en comparación a los prácticos, los estudiantes perciben una mayor exposición a prácticas generativas centradas en la figura del profesor. Por ejemplo, ver al profesor explicar conceptos de la asignatura, o explicitar sus propios procesos de pensamiento. En los cursos didácticos y prácticos, en contraste con los disciplinares, los estudiantes perciben mayores oportunidades para participar en discusiones productivas. Finalmente, en los cursos didácticos se perciben mayores oportunidades para realizar tareas desafiantes que en los cursos disciplinares.

\subsubsection{Análisis de caso y modelamiento o demostraciones}

¿Cuáles son las diferencias entre cursos didácticos y prácticos en relación a oportunidades para ver modelamiento de practicas y generativas y analizar casos?

Como muestra la tabla 6 , los estudiantes perciben mayores oportunidades para ver al formador demostrando prácticas pedagógicas en los cursos de didáctica que en los de práctica (significativo en $\mathrm{p}<0,05$ ). Asimismo, para los cursos didácticos perciben oportunidades significativamente mayores para ver videos de enseñanza en el aula y trabajar con materiales didácticos reales.

Para los cursos prácticos, en comparación a los didácticos, los estudiantes perciben mayores oportunidades para analizar casos a la luz de la teoría y observar la puesta en práctica de métodos o estrategias de enseñanza (ambas diferencias significativas en $\mathrm{p}<0.01$ ).

Tabla 6. Promedio, desviación estándar y comparaciones por tipo de curso

\begin{tabular}{|c|c|c|c|c|}
\hline & Didácticas & Prácticas & $\mathrm{T}$ & Sig \\
\hline $\begin{array}{l}\text { Analizar casos o episodios de interacción en sala } \\
\text { de clases a la luz de modelos o teorías revisadas } \\
\text { en este curso }\end{array}$ & $3,08(0,87)$ & $3,66(0,59)$ & $-7,19 * *$ & 0,00 \\
\hline $\begin{array}{l}\text { Analizar o trabajar con planificaciones o materiales } \\
\text { didácticos reales }\end{array}$ & $3,40(0,77)$ & $3,17(0,86)$ & $2,40 *$ & 0,02 \\
\hline $\begin{array}{l}\text { Examinar transcripciones de conversaciones reales } \\
\text { de clases escolares o discusiones de estudiantes }\end{array}$ & $2,30(1,08)$ & $2,35(1,21)$ & $-0,38$ & 0,70 \\
\hline $\begin{array}{l}\text { Hacer trabajos, solucionar problemas, estudiar o } \\
\text { leer textos, que tus propios estudiantes tendrán que } \\
\text { hacer, para verlos desde su perspectiva }\end{array}$ & $3,14(0,94)$ & $3,20(0,90)$ & $-0,45$ & 0,65 \\
\hline $\begin{array}{l}\text { Observar directamente o a través de un video, } \\
\text { cómo un profesor pone en práctica una estrategia o } \\
\text { método de enseñanza vista en este curso }\end{array}$ & $2,60(1,08)$ & $3,34(0,89)$ & $-6,45 * *$ & 0,00 \\
\hline Observar o analizar videos de enseñanza en el aula & $2,59(1,11)$ & $2,27(1,31)$ & $2,27 *$ & 0,02 \\
\hline $\begin{array}{l}\text { Ver a tu profesor o formador haciendo una } \\
\text { demostración de alguna de las prácticas } \\
\text { pedagógicas estudiadas en el curso }\end{array}$ & $3,02(0,93)$ & $2,42(1,01)$ & $4,95^{* *}$ & 0,00 \\
\hline $\begin{array}{l}\text { Analizar o discutir cómo los contenidos teóricos } \\
\text { permiten enfrentar necesidades concretas de la } \\
\text { práctica pedagógica en la escuela }\end{array}$ & $3,42(0,72)$ & $3,34(0,65)$ & 0,99 & 0,32 \\
\hline
\end{tabular}

$* \mathrm{p}<0,05 ; * * \mathrm{p}<0,01$ 


\subsubsection{Aproximación a nivel de ensayo}

\subsubsection{Ensayos o simulaciones en los cursos universitarios}

¿Cuál es la diferencia entre cursos didácticos y prácticos en las oportunidades de aprendizaje percibidas por los estudiantes para ensayar prácticas generativas en los cursos universitarios?

Solo se observaron diferencias significativas en las oportunidades para ensayar planificar la enseñanza. Los estudiantes perciben que ellas son significativamente mayores $(\mathrm{p}<0,01)$ en los cursos prácticos $(\mathrm{M}=3,85 ; \mathrm{DE}=0,42)$ que para los didácticos $(\mathrm{M}=3,28$; $\mathrm{DE}=0,94)$.

En la parte II del cuestionario se preguntó a los estudiantes por sus oportunidades para ensayar prácticas generativas específicas en sus clases de didáctica y de práctica. Como ilustra el gráfico 1, los resultados fueron similares para cursos didácticos y prácticos: para la mayoría de las prácticas generativas, menos de la mitad de los estudiantes (porcentaje $\leq 50 \%$ ) señaló contar con oportunidades para ensayarlas en las clases en la universidad.

Gráfico 1. Porcentajes de frecuencia para oportunidades de ensayo de prácticas generativas en cursos de didáctica y práctica

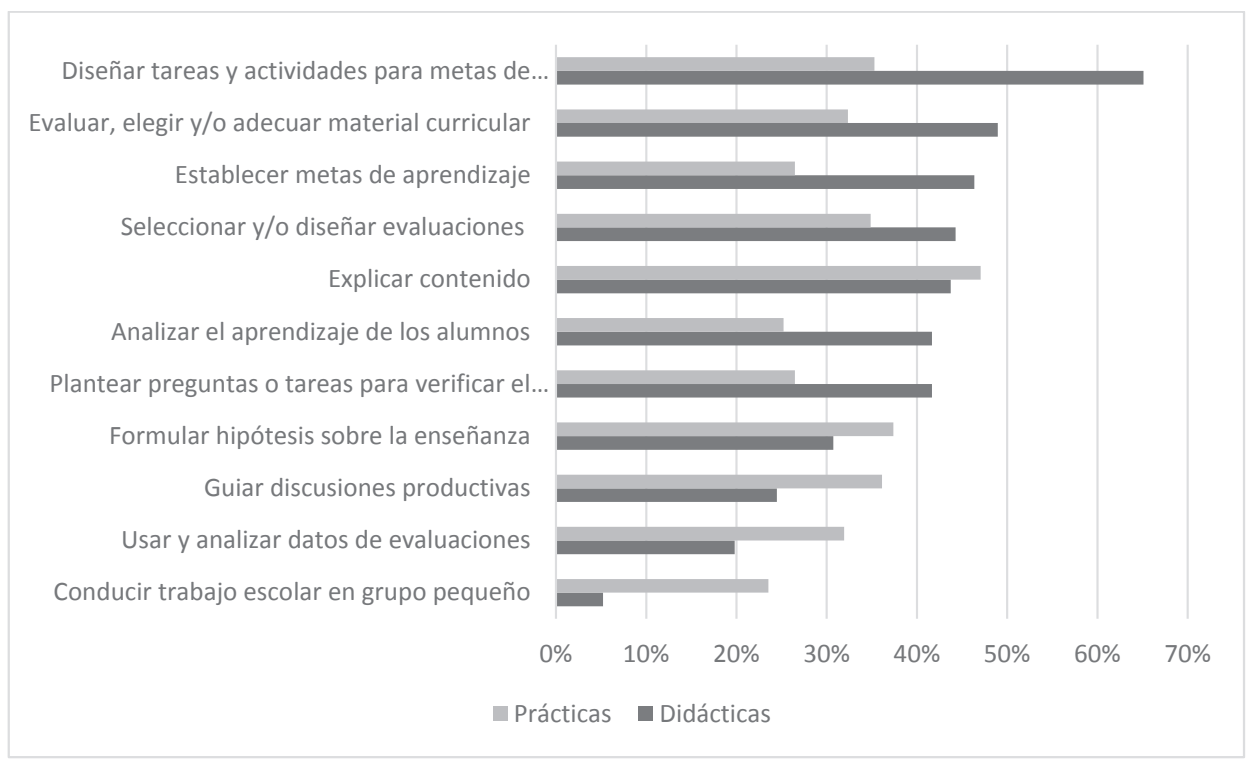

Fuente: Elaboración propia

En los cursos didácticos, los estudiantes percibieron mayores oportunidades para ensayar "diseñar tareas y actividades para metas de aprendizaje" (65\%) y "evaluar, elegir y/o adecuar material curricular" (49\%). Por el contrario, percibieron menores oportunidades 
para ensayar "conducir trabajo escolar en grupo pequeño" (5\%) y "usar y analizar datos de evaluaciones" (20\%).

Para los cursos prácticos, los estudiantes percibieron mayores oportunidades para ensayar "explicar un contenido" (47\%) y "formular hipótesis sobre la enseñanza" (37\%). Por el contrario, percibieron menores oportunidades para "conducir trabajo escolar en grupo pequeño" (24\%) y "analizar el aprendizaje de los alumnos" (26\%).

5.2.2.2. Experiencia directa de prácticas generativas en la sala de clases

¿Cuáles son las diferencias en las experiencias directas de los estudiantes según el tipo de práctica realizada?

En los cursos prácticos se indagó en las oportunidades para desplegar las prácticas generativas en el contexto escolar, con el fin de poder detectar si a partir de la percepción de los estudiantes en las distintas instancias se podía distinguir una progresión. Primero, en el gráfico 2 se muestran las diferencias en las oportunidades de tener experiencias directas en aula para cada práctica generativa, según el tipo de práctica realizada: práctica 1 (primer año), práctica 2 (tercer año) y práctica 3 (cuarto año). Para todas las prácticas generativas consultadas se observan mayores oportunidades de experiencia directa en la práctica 3.

Gráfico 2. Porcentajes de frecuencia para oportunidades de ensayo de prácticas generativas, por tipo de práctica realizada

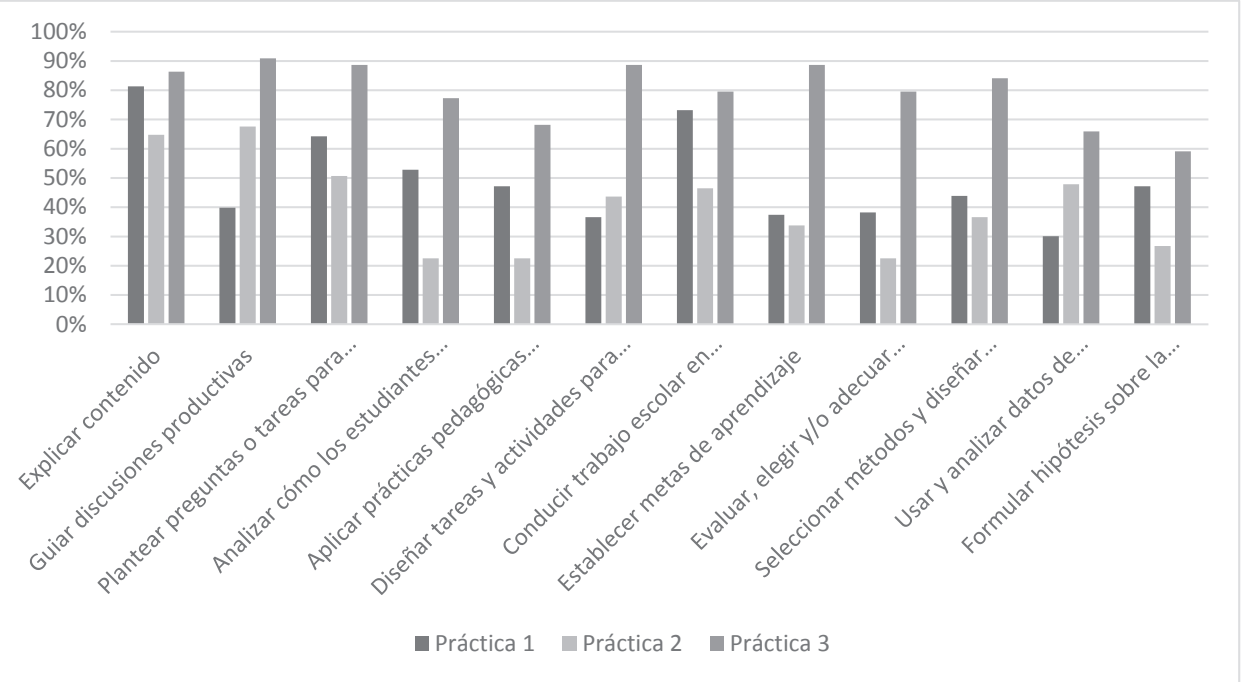

Segundo, en el gráfico 3 se muestran las diferencias en aproximaciones a la práctica, según el tipo de práctica realizada (práctica 1, 2 o 3), en relación a la cantidad de alumnos de los que se tenían que hacer cargo. Los estudiantes perciben pocas oportunidades para ensayar prácticas generativas con un niño en forma individual en todos los tipos de práctica (rango entre $9 \%$ y 17\%). En la práctica 1 se perciben las mayores oportunidades para 
ensayar prácticas generativas con un grupo pequeño (38\%). Al contrario, las menores oportunidades para ensayar con grupo pequeño se perciben para la práctica $2(11 \%)$. Para el ensayo de prácticas generativas con el curso completo, las mayores oportunidades se percibieron en la práctica $2(34 \%)$ y en la $3(37 \%)$.

Gráfico 3. Porcentajes de frecuencia para aproximaciones a la práctica por curso de práctica

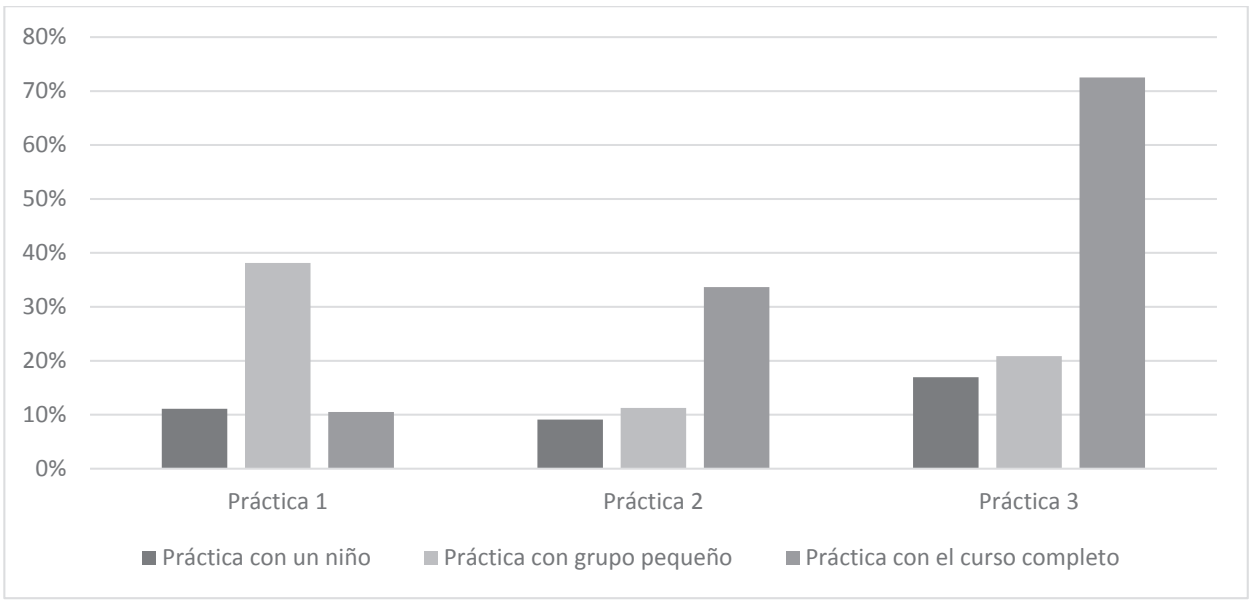

\section{DISCUSIONES}

La aplicación de este cuestionario, que indaga en las oportunidades de aprendizaje en la formación inicial, ha permitido conocer la percepción de los estudiantes en un programa universitario de formación de profesores. Tal como fue descrito en los antecedentes del estudio, este programa se encuentra en un proceso de rediseño hacia un currículum basado en la práctica y, este instrumento, fue diseñado con el objetivo de levantar una línea de base para guiar dicho proceso de cambio y ajustar el rediseño en base a evidencias.

Con base en el marco teórico de coherencia (Hammerness \& Klette, 2015; Hammerness, 2006; Darling-Hammond et al., 2005), el cuestionario examina la percepción que los estudiantes tienen respecto a una visión común de la enseñanza-aprendizaje, la alineación entre los cursos a lo largo del programa, y la conexión entre las clases al interior de un mismo curso. Al respecto, la percepción de coherencia de los estudiantes fue menor para los cursos disciplinares en comparación a los de didáctica y práctica, en los cuales se observó una percepción de coherencia similar. La conexión del curso disciplinar con otros cursos del programa de formación es el ámbito percibido con una menor coherencia. Esto se podría explicar considerando que los cursos disciplinares, tradicionalmente, no han estado articulados con los otros cursos del programa dado que están a cargo de las facultades disciplinares. En el actual rediseño curricular, se ha puesto especial énfasis en mejorar la articulación entre las facultades disciplinares y los programas de formación, con el fin de impactar en la coherencia percibida por los estudiantes. Es de esperar entonces 
que, una vez implementados los cambios curriculares diseñados, se observe un incremento en esta percepción de coherencia de los cursos disciplinares, por parte de los estudiantes.

Considerando el marco de pedagogías de la práctica (Grossman et al., 2009), este instrumento evalúa las oportunidades percibidas por los estudiantes para aprender prácticas generativas específicas. En ese sentido, en relación a las representaciones de la práctica, los estudiantes perciben menos oportunidades para ver modelos de enseñanza -acerca del uso de representaciones, ejemplos o modelos para explicar los conceptos, a la explicitación de los propios procesos de pensamiento, y a la relación de tareas desafiantes- en los cursos de práctica que en los disciplinares y didácticos. En cambio, en relación a participar en discusiones productivas, perciben más oportunidades en los cursos de práctica. Dado lo anterior, se esperaría que una vez consolidados los cambios curriculares, los supervisores de práctica incluyan intencionadamente estas prácticas generativas en su docencia universitaria, de tal manera que los estudiantes estén inmersos en un contexto donde estas prácticas generativas se ponen en acto. Por otra, parte se esperaría que en aplicaciones futuras aumentaran las oportunidades de que los estudiantes pudiesen discutir y argumentar en los cursos disciplinares de tal manera que pudiesen observar buenos modelos de enseñanza de la disciplina y también de lograr aprendizajes más profundos.

También a nivel representacional, las oportunidades percibidas por los estudiantes, tanto en los cursos de didáctica como de práctica, sugieren mayores oportunidades para representar la práctica a través de material teórico o del diseño de una clase -como casos de enseñanza en el aula, trabajo con materiales didácticos, discusiones teóricas o análisis de la práctica a la luz de la teoría- que para representarse la práctica a través de evidencias reales de clases implementadas en el aula, como videos, transcripciones o evaluaciones de estudiantes. Ello plantea el doble desafío de avanzar en la sistematización de evidencias reales de clases y de promover su uso por parte de los formadores. Iniciativas previas han sido desarrolladas para sistematizar evidencias de clases reales. La elaboración de una videoteca que recoge prácticas ejemplares en lenguaje y matemáticas en segundo ciclo, y las pone a disposición mediante videoclips, se enmarca en esta línea (Müller, Calcagni, Grau, Preiss \& Volante, 2013). Sin embargo, en otras asignaturas y ciclos aún es difícil acceder a material de este tipo, y la mayor parte de este material no está desarrollado en el contexto nacional y tampoco está disponible en español. Asimismo, son escasas las transcripciones de clases o evidencias reales del aprendizaje de alumnos. Sistematizar material de este tipo sin duda es un desafío que aportaría al desarrollo de las pedagogías de la práctica en formación inicial.

Uno de los desafíos que plantea el desarrollar un currículum basado en la práctica es incorporar a la docencia universitaria oportunidades de aproximación sucesiva a la práctica (Grossman et al., 2009), de tal manera que los estudiantes tengan oportunidades previas al desempeño en el contexto de máxima complejidad, que es el aula escolar. En este sentido, contar con evidencias reales -ya sea videos, transcripciones, tareas, textos, evaluaciones, etc.- es un recurso indispensable. Al introducir las prácticas generativas se hace necesario incluirlos de forma sistemática a la docencia universitaria de la formación inicial. Por otra parte, es fundamental considerar estos materiales no como un fin en sí mismos, sino como parte de un ciclo en el que se introducen las prácticas generativas, luego se preparan y ensayan, para posteriormente desplegarlas en la sala de clase, ya sea a cargo de un curso o en co-docencia, para terminar el ciclo analizando los registros de la propia práctica con el propósito de mejorarla (McDonald, Kazemi \& Schneider, 2013). Considerando 
los resultados, se evidencia el desafío de lograr una coordinación entre la didáctica y la práctica en este ciclo, donde la didáctica ponga más énfasis en la introducción y ensayos en relación a prácticas generativas específicas, y en la práctica se asegure que estas prácticas generativas se incluyen tanto en el desempeño en aula como en el análisis de la propia práctica. Este análisis implica, por otra parte, tener acceso a filmar los desempeños de los estudiantes en sus respectivos contextos de práctica lo que supone una coordinación con los centros en relación a las autorizaciones y consentimientos que esta actividad implica para cumplir con los requerimientos éticos. Esto es especialmente relevante considerando que la observación de videos de la propia práctica aparece en la literatura de formación inicial como una estrategia de autoevaluación que tiene impacto en el mejoramiento del desempeño (Rosaen, Lundeberg, Cooper, Fritzen \& Terpstra, 2008; Sewall, 2009).

Por otra parte con el propósito de obtener información en relación a cómo perciben los estudiantes las aproximaciones sucesivas a las prácticas generativas, el cuestionario indaga en las oportunidades para ensayar o simular la enseñanza en el aula, antes de la práctica misma con estudiantes en el contexto escolar. Estas oportunidades refieren a "poner en acto" las diferentes prácticas generativas, como planificar la enseñanza, establecer metas de aprendizaje, explicar contenido y guiar discusiones productivas en el contexto de los cursos universitarios. En relación con ello, los resultados de percepción de los estudiantes, tanto para los cursos de didáctica como de práctica, dan cuenta que las oportunidades de ensayo se concentran mayoritariamente en actividades de preparación para la enseñanza como el diseño de material curricular, la planificación de una clase o segmento de clase y el establecimiento de metas de aprendizaje. Sólo en los cursos de práctica se perciben mayores oportunidades para ensayar la explicación de un contenido. Considerando las fases de la enseñanza preactiva (es decir, sin presencia de alumnos) e interactiva (con alumnos), descritas por Jackson (1968), las oportunidades de aprendizaje percibidas se concentrarían principalmente en la fase pre-activa, específicamente en la planificación de la enseñanza. Esto coincide con lo planteado por Grossman et al., (2009), en relación al énfasis que los programas de formación inicial ponen en la planificación como principal forma de aproximación a la práctica. En relación a las menores oportunidades percibidas, estas se concentran en las actividades propias de la interacción con los alumnos, como conducir trabajo escolar en grupo pequeño o aplicar prácticas pedagógicas efectivas. En esta línea, tras el trabajo de rediseño hacia un currículum basado en la práctica, se esperaría que los estudiantes perciban cada vez mayores oportunidades para ensayar o simular las prácticas generativas, tanto en sus cursos de didáctica como de práctica.

Las experiencias de práctica en el contexto escolar son la aproximación más directa a la práctica profesional docente y, también, la más compleja. Para ellas, el cuestionario indaga tanto en las oportunidades para aplicar prácticas generativas específicas (por ejemplo, evaluar, elegir y/o adecuar material curricular, guiar discusiones productivas, etc.), como en el alcance de dichas prácticas, es decir, si fueron aplicadas con un solo niño, con un grupo pequeño de niños, o con un curso completo. Para los cursos de didáctica, los estudiantes perciben escasas oportunidades de experiencia directa de práctica. En los cursos de práctica, por el contrario, perciben una mayor cantidad de experiencias en este sentido, destacando las oportunidades para aplicar prácticas generativas vinculadas a la fase interactiva (Jackson, 1968), como explicar contenido y guiar discusiones productivas. Aquellas prácticas generativas percibidas con menores oportunidades de aprendizaje, en los cursos de práctica, tienden a vincularse con la selección y/o adecuación de material 
Estudios Pedagógicos XLII, N 4: 145-163, 2016

PERCEPCIÓN DE ESTUDIANTES DE PEDAGOGÍA EN RELACIÓN A LAS OPORTUNIDADES PARA EL DESARROLLO DE PRÁCTICAS GENERATIVAS EN SU FORMACIÓN

curricular y con la evaluación de los aprendizajes. Considerando lo anterior, la línea de base plantea el desafío de brindar a los futuros profesores mayores oportunidades para vincular la enseñanza de contenidos con la preparación de material curricular pertinente y la evaluación de los aprendizajes esperados.

Coherente con las nociones del modelo de cambio desarrolladas en el marco teórico de este estudio, el cuestionario permite levantar una línea base para contar con evidencia que informe la intervención en las áreas de interés para el rediseño curricular. Además del levantamiento de la línea de base, el instrumento constituye una herramienta de mejora continua, en la medida que permite monitorear los resultados del proceso de rediseño, permitiendo la toma de decisiones basadas en evidencia. Se considera que la experiencia de aplicación del cuestionario en el programa, en conjunto con otras líneas del trabajo de rediseño, también ha favorecido la instalación gradual de un vocabulario y expectativas comunes entre académicos y estudiantes.

Dentro de las limitaciones del estudio cabe destacar que la aplicación del instrumento se realizó en todos los cursos rediseñados. Esto implicó en muchos casos que estudiantes lo tuviesen que responder para tres o cuatro cursos en el semestre, lo que significó un cierto nivel de desgaste por parte de los mismos, pudiendo afectar la precisión de sus respuestas. En el futuro se evaluará la posibilidad de aplicar el instrumento por grupos de cursos, con el fin de avanzar hacia un proceso más parsimonioso.

Si bien el instrumento constituye un avance en relación al monitoreo del mecanismo de cambio propuesto para el rediseño curricular, se hace necesario complementar la información entregada por éste con entrevistas o grupos focales a estudiantes, profesores, colaboradores y académicos, además de incluir el análisis de los desempeños de los estudiantes en las tareas principales de los cursos y la observación de clases, como propone el modelo de evaluación del estudio CATE (Hammerness \& Klette, 2015), en el cual se originan parte de los constructos que sirvieron de base para la elaboración de este instrumento.

Por otra parte, para que la información entregada por el instrumento impacte en las prácticas de docencia universitaria, debe acompañarse de apoyos que faciliten el avance de los académicos en la dirección propuesta. Para esto se está avanzado en la elaboración de una matriz en que, para cada práctica generativa, se sugieren actividades para cuatro diferentes niveles de aproximación a la práctica: (1) observación o modelaje, (2) análisis de casos, (3) ensayo en clases, y (4) experiencia directa en aula. El objetivo de esta matriz es ser una guía para los formadores que permita avanzar en la incorporación de las pedagogías de la práctica a la formación inicial.

\section{REFERENCIAS BIBLIOGRÁFICAS}

Ball, D. \& Forzani, F. (2009). The work of Teaching and the Challenge for Teacher Education. Journal of Teacher Education, 60 (5), 497-511. doi: 10.1177/0022487109348479

Center to Support Excellence in Teaching - CSET (s/f). Core Teaching Practices - Delphi Studies. Recuperado de https://cset.stanford.edu/research/core-practices

Darling-Hammond, L. (2000). How Teacher Education matters. Journal of Teacher Education, 51(3), 166- 173. doi: 10.1177/0022487100051003002

Darling-Hammond, L., Hammerness, K., Grossman, P., Rust, F. \& Shulman, L. (2005). The design of teacher education programs. En L. Darling-Hammond \& J. Bransford (Eds.). Preparing teachers 
for a changing world (pp. 390-441). San Francisco: Jossey-Bass.

Davis, E. \& Boerst, T. (2014). Designing elementary teacher education to prepare well-started beginners. Recuperado de http://www.teachingworks.org/research-data/workingpapers

Forzani, F. (2014). Understanding "Core Practices" and "Practice-Based" Teacher Education: Learning from the Past. Journal of Teacher Education, 65(4), 357-368. doi: 10.1177/0022487114533800

Grossman, P., Compton, C., Igra, D., Ronfeldt, M., Shahan, E. \& Williamson, P. (2009). Teaching Practice: A Cross-Professional Perspective. Teachers College Record, 111(9), 2055-2100.

Grossman, P., Hammerness, K. \& McDonald, M. (2009). Redefining teaching, re-imagining teacher education. Teachers and Teaching: theory and practice, 15(2), 273-289. doi: $10.1080 / 13540600902875340$

Hammerness, K. \& Klette, K. (2015). Indicators of Quality in Teacher Education: Looking at Features of Teacher Education from an International Perspective. To appear in Gerald LeTendre \& Alexander Wiseman (Eds). Promoting and Sustaining a Quality Teaching Workforce. England: Emerald Press.

Hammerness, K. (2006). From Coherence in Theory to Coherence in Practice. Teachers College Record, 108(7), 1241-1265.

Hanushek, E. (1992). The trade -off between child quantity and quality. Journal of Political Economy, 100(1), 84-117.

Jackson, P. (1965). The way teaching is. Washington: National Educational Association.

Mc Donald, M., Kazemi, E. \& Schneider, S. (2013). Core Practices and Pedagogies of Teacher Education: A Call for a Common Language and Collective Activity. Journal of Teacher Education, XX(X), 1-9. doi: 10.1177/0022487113493807

Ministerio de Educación (2011). Estándares orientadores para egresados de carreras de Pedagogía en Educación Básica. Santiago de Chile: Ministerio de Educación. Disponible en: http:// bibliorepo.umce.cl/libros_electronicos/basica/egba_1.pdf

Müller, M., Calcagni, E., Grau, V., Preiss, D. \& Volante, P. (2013). Desarrollo de habilidades de observación en estudiantes de pedagogía: Resultados de una intervención piloto basada en el uso de la Videoteca de Buenas Prácticas Docentes. Estudios Pedagógicos, XXXIX (1), 85-113. doi:10.4067/s0718-07052013000300007

Müller, M. (en prensa). Aproximaciones sucesivas al desempeño auténtico: Sistema de Prácticas Pedagógicas UC. En L. Meckes \& C. Cox (Eds) Formación de Profesores en Chile: Nudos y Oportunidades para su transformación. CEPPE: Universidad Católica.

OREAL/UNESCO (2013). Catastro de experiencias relevantes de políticas docentes en América Latina. Recuperado de http://www.politicasdocentesalc.com/index.php/productos-esperados

Organization for Economic Cooperation and Cultural Development (OECD) (2005). Teachers matter: Attracting, developing and retaining effective teachers. Paris: OECD.

Rivers, J. \& Sanders, W. (1996). Cumulative and residual effects of teachers on future student academic achievement. Knoxville: University of Tennesse Value-Added Research and Assessment Center.

Rosaen, C.L., Lundeberg, M., Cooper, M., Fritzen, A. \& Terpstra, M. (2008). Noticing noticing: How does investigation of video records change how teachers reflect on their experiences? Journal of teacher Education, 59(4), 347-360. doi: 10.1177/0022487108322128

Sewall, M. (2009). Transforming supervision: Using video elicitation to support preservice teacherdirected reflective conversations. Issues in Teacher Education, 18(2), 11-30. 
